\title{
Efeito da vitamina D3 e 25-hidroxi-colecalciferol sobre o desempenho, o rendimento de carcaça e a morfologia intestinal de frangos de corte ${ }^{1}$
}

\author{
Jerônimo Ávito Gonçalves de Brito², Antônio Gilberto Bertechini ${ }^{3}$, Édison José Fassani ${ }^{3}$ \\ Paulo Borges Rodrigues ${ }^{3}$, Eduardo Machado Costa Lima ${ }^{4}$, Camila Meneghetti ${ }^{4}$
}

\author{
1 Projeto financiado pela FAPEMIG. \\ 2 Doutorando do Programa de Pos-Graduação em Zootecnia da UFLA. \\ ${ }^{3}$ Departamento de Zootecnia da UFLA. \\ ${ }^{4}$ Acadêmico do NECTA-Núcleo de Estudos em Ciência e Tecnologia Avícola da UFLA.
}

RESUMO - O experimento foi conduzido com o objetivo de avaliar níveis de suplementação de vitamina D proveniente de duas fontes $\left(\mathrm{D}_{3}\right.$ e $\left.25-\mathrm{OHD}_{3}\right)$ em rações para frangos de corte criados em gaiolas. Foram utilizados 1.500 pintos machos de 1 dia, linhagem Cobb-700, alojados em gaiolas metálicas (100) com dispositivos e manejo adaptados para criação das aves. Em cada fase do desenvolvimento (inicial, de 1 a 21 dias; de crescimento, 22 a 38 dias; e final, de 39 a 45 dias), avaliaram-se quatro níveis (programas) de suplementação de vitamina D (20/16/10; 37,5/30/18,8; 87,5/70/43,8 e 137,5/110/68,8 $\mu \mathrm{g} / \mathrm{kg}$ ração), oriunda de duas fontes. Os dois tratamentos adicionais foram constituídos da combinação das duas fontes $\left(\mathrm{D}_{3}+25-\mathrm{OHD}\right)_{3}$, em diferentes proporções $(50+37,7 / 40+30 / 25+18,8$ e $50+70 / 40+56 / 25+35 \mu \mathrm{g} / \mathrm{kg}$ de ração para as fases inicial, crescimento e final, respectivamente). O delineamento experimental foi inteiramente casualizado, em esquema fatorial $2 \times 4+2$ (fontes × níveis + adicionais) e 10 repetições por tratamento As rações foram à base de milho e farelo de soja, com suplementação de fitase $(500 \mathrm{ftu} / \mathrm{kg})$, segundo recomendações das tabelas brasileiras de exigências nutricionais de animais não-ruminantes. Foram avaliados o desempenho, o rendimento de carcaça e a morfologia intestinal das aves. A suplementação de vitamina D na fase inicial melhorou o ganho de peso e a conversão alimentar das aves. Maior ganho de peso e consumo de ração aos 45 dias foi observado nas aves dos tratamentos adicionais (associações). O rendimento de carcaça aumentou com o uso de $25-\mathrm{OHD}_{3}$. Em comparação à suplementação isolada das fontes de vitamina $\mathrm{D}$, os tratamentos adicionais promoveram maiores benefícios para a maioria das características avaliadas. A adição de $25-\mathrm{OHD}_{3}$ em rações contendo vitamina $\mathrm{D}_{3}$ melhora as características de desempenho dos frangos de corte.

Palavras-chave: fontes de vitamina $\mathrm{D}$, metabólito de vitamina $\mathrm{D}_{3}$, vitamina $\mathrm{D}$

\section{Effect of vitamin $D_{3}$ and 25-hidroxicholecalciferol on performance, carcass yield and intestinal morphology in broiler chickens}

\footnotetext{
ABSTRACT - The experiment was conducted aiming at evaluating the levels of supplementation of vitamin D from two sources (vitamin $\mathrm{D}_{3}$ and $25-\mathrm{OHD}_{3}$ ) on diets for broiler chickens raised in cages. It was used 1,500 line Cobb-700 male chicks at one day of age, housed in metal cages (100), with appliances and management fitted for bird raising. In each growing phase (starter, from 1 to 21 days; growing, from 22 to 38 days, and finishing, from 39 to 45 days), it was evaluated four levels (programs) of vitamin D supplementation $(20 / 16 / 10 ; 37.5 / 30 / 18.8 ; 87.5 / 70 / 43.8$ and $137.5 / 110 / 68.8 \mu \mathrm{g} / \mathrm{kg}$ ration) from two sources. The two additional treatments consisted of combination of the two sources $\left(\mathrm{D}_{3}+25-\mathrm{OHD}_{3}\right)$ at different proportions $(50+37.7 / 40+30 / 25+18.8$ and $50+70 / 40+56 / 25+35 \mu \mathrm{g} / \mathrm{kg}$ ration for starter, growing and finishing phases, respectively). It was used a complete random experimental design, in a $2 \times 4+2$ (sources $\times$ levels + aditionals) and 10 replicates per treatment. The diets were based on corn and soybean meal, with supplementation of phytase $(500 \mathrm{ftu} / \mathrm{kg})$, following the recommendations of the national tables of nutritional requirements of non-ruminant animals. The performance and carcass characteristics as well as the intestinal morphology of the birds were evaluated. Vitamin-D supplementation at the starter phase improved weigh gain and feed conversion of the birds. Greater weight gain and feed intake at 45 days of age were observed for the group of birds of the additional treatment associations. Carcass yield increased with the use of $25-\mathrm{OHD}_{3}$. When compared to the single supplementation of vitamin D sources, the additional treatments promoted more benefits for most of the evaluated characteristics. The addition of 25-OHD3 in rations containing vitamin D3 improves performance characteristics of broilers
}

Key Words: metabolites of vitamin $\mathrm{D}_{3}$, vitamin $\mathrm{D}$, vitamin $\mathrm{D}$ sources 


\section{Introdução}

A vitamina D tem participação importante no metabolismo ósseo, e é diretamente responsável pelo crescimento esquelético que dá suporte para que as aves possam obter o seu máximo desempenho produtivo. A má-formação óssea é um dos fatores que dificultam a ingestão de alimento, prejudicando o ganho de peso dos frangos de corte.

Alguns trabalhos indicam que o desempenho das aves não é afetado pela suplementação de fontes de vitamina $D$ (Edwards, 2002; Ledwaba et al., 2003; Korver, 2005), enquanto outros (Yarger et al., 1995; Fritts \& Waldroup, $2003,2005)$ indicam efeito benéfico do uso do metabólito $25 \mathrm{OHD}_{3}$.

Há relatos a campo sobre a incidência de sinais de hemorragia ao longo do intestino de aves alimentadas com a fonte $25-\mathrm{OHD}_{3}$ ou com altos níveis de inclusão de vitamina $\mathrm{D}_{3}$. Também existem trabalhos sobre a inter-relação do metabolismo das vitaminas lipossolúveis que, em alguma extensão, estão associadas ao bom desenvolvimento das células intestinais, principalmente as vitaminas A e E que, porventura, poderiam ser metabolicamente afetadas pela maior ou menor atividade e absorção da vitamina $\mathrm{D}$ dietética.

São escassas as pesquisa sobre a suplementação de vitamina D no rendimento de carcaça e dos cortes de frangos de corte. Korver (2005) observou maiores rendimentos de carcaça e de peito em frangos de corte aos 42 dias de idade quando suplementou vitamina $\mathrm{D}_{3}(2.500 \mathrm{UI} / \mathrm{kg})$ em conjunto com $25-\mathrm{OHD}_{3}(69 \mu \mathrm{g}$ ou $2.760 \mathrm{UI} / \mathrm{kg}$ de 1 a 21 dias $)$. Esse autor verificou também maior densidade cortical das tíbias provenientes dos frangos alimentados continuamente com $25-\mathrm{OHD}_{3}(2.760 \mathrm{UI} / \mathrm{kg})$ e redução da incidência de black bone.

Apesar do grande número de estudos, ainda não existe consenso sobre os efeitos de níveis e associação de fontes de suplementação de vitamina D nas rações sobre o desempenho, as características de carcaça e a morfologia intestinal de frangos de corte.

Assim, pretendeu-se com este estudo avaliar a utilização do metabólito 25-hidroxi-colecalciferol $\left(\mathrm{OHD}_{3}\right) \mathrm{em}$ comparação à forma tradicional de suplementação (vitamina $\mathrm{D}_{3}$ - colecalciferol) em rações para frangos de corte e determinar seus efeitos sobre o desempenho, o rendimento de carcaça e a morfologia intestinal dessas aves.

\section{Material e Métodos}

Foram utilizados 1.500 pintos machos de 1 dia, linhagem Cobb-700, alojados em 100 gaiolas de metabolismo com $0,473 \mathrm{~m}^{2}$ cada, em instalação com ambiente semicontrolado. A iluminação foi contínua nas 24 horas e o aquecimento nos primeiros dez dias foi realizado com lâmpadas incandescentes de $150 \mathrm{~W}$, instaladas no interior das gaiolas. Adotou-se um delineamento inteiramente casualizado, em esquema fatorial $2 \times 4+2$, composto de duas fontes de suplementação de vitamina $\mathrm{D}\left(\mathrm{D}_{3}\right.$ e $\left.25-\mathrm{OHD}_{3}\right)$, quatro níveis de suplementação e duas associações das fontes de vitamina $\mathrm{D}$, totalizando dez tratamentos, cada um com dez repetições.

Os tratamentos experimentais (Tabela 1) foram constituídos de quatro níveis de suplementação de vitamina $\mathrm{D}(20,0 ; 37,5 ; 87,5 ; \mathrm{e} 137,5 \mu \mathrm{g} / \mathrm{kg}$ de ração), provenientes das duas fontes de suplementação, e mais dois tratamentos adicionais, constituídos pela combinação das duas fontes em diferentes proporções $\left(50,0 \mathrm{ig} / \mathrm{kg} \mathrm{de} \mathrm{D}_{3}+37,5 \mu \mathrm{g} / \mathrm{kg}\right.$ de $25-\mathrm{OH} \mathrm{D}_{3}$ e $50,0 \mu \mathrm{g} / \mathrm{kg}$ de $\mathrm{D}_{3}+70,0 \mathrm{ig} / \mathrm{kg}$ de $25-\mathrm{OH} \mathrm{D} \mathrm{D}_{3}$ ). A suplementação de vitamina $\mathrm{D}$ foi reduzida gradativamente na fase de crescimento $(20 \%)$ e na fase final $(50 \%) \mathrm{em}$ relação à fase inicial. A parcela experimental foi constituída de 15 aves no período de 1 a 21 dias; oito aves no período

Tabela 1 - Níveis e fontes de suplementação de vitamina D nas três fases de desenvolvimento

\begin{tabular}{|c|c|c|}
\hline Fase inicial & Fase crescimento ( $20 \%$ de redução) & Fase final $(50 \%$ de redução $)$ \\
\hline & \multicolumn{2}{|c|}{ Vitamina $D_{3}(\mu \mathrm{g} / \mathrm{kg})$} \\
\hline 20,0 & 16,0 & 10,0 \\
\hline 37,5 & 30,0 & 18,8 \\
\hline \multirow[t]{2}{*}{137,5} & 110,0 & 68,8 \\
\hline & \multicolumn{2}{|c|}{ 25-hidroxi-colecalciferol $(\mu \mathrm{g} / \mathrm{kg})$} \\
\hline 87,5 & 70,0 & 43,8 \\
\hline \multirow[t]{2}{*}{137,5} & 110 & 68,8 \\
\hline & \multicolumn{2}{|c|}{ Vit. $D_{3}+25$-hidroxi-colecalciferol $(\mu \mathrm{g} / \mathrm{kg})$} \\
\hline $50+37,5$ & $40,0+30,0$ & $25,0+18,8$ \\
\hline $50+70,0$ & $40,0+56,0$ & $25,0+35,0$ \\
\hline
\end{tabular}


de 22 a 38 dias; e seis aves no período de 39 a 45 dias de idade, para ajustes da densidade de criação.

As rações experimentais (Tabela 2) eram à base de milho e farelo de soja, de acordo com o programa alimentar, composto por três rações (inicial 1 a 21 dias; crescimento 22 a 38 dias e final 39 a 45 dias), seguindo as recomendações de Rostagno et al. (2005), com suplementação de fitase em todas as rações (Ronozyme P CT - $500 \mathrm{ftu} / \mathrm{kg}$ ) e redução de $25 \%$ no teor de fósforo disponível e $10 \%$ nas exigências dietéticas de cálcio (Shoulten et al., 2003).

A fonte de vitamina $\mathrm{D}_{3}$ utilizada foi proveniente da DSM Produtos Nutricionais do Brasil (DSM), contendo $500 \mathrm{mg} / \mathrm{kg}(20.000 .000 \mathrm{UI} / \mathrm{kg})$ e a fonte de $25-\mathrm{OHD}_{3}$ $69 \mathrm{mg} / \mathrm{kg}(2.760 .000 \mathrm{UI} / \mathrm{kg})$ de suplemento. As rações experimentais, para cada fase, foram isonutritivas, com exceção dos níveis de vitamina $\mathrm{D}$, que constituíram os tratamentos e foram preparadas e armazenadas em sala

Tabela 2 - Composição percentual e níveis nutricionais calculados das rações experimentais

\begin{tabular}{|c|c|c|c|}
\hline$\overline{\text { Ingrediente }(\%)}$ & Inicial & Crescimento & Final \\
\hline Milho & 55,067 & 60,982 & 64,904 \\
\hline Farelo de soja & 36,329 & 30,315 & 26,816 \\
\hline Fosfato bicálcico & 1,285 & 1,064 & 0,899 \\
\hline Calcário calcítico & 0,955 & 0,895 & 0,845 \\
\hline Óleo de soja & 4,817 & 5,229 & 5,192 \\
\hline Sal comum & 0,457 & 0,425 & 0,402 \\
\hline DL-metionina $99 \%$ & 0,240 & 0,222 & 0,197 \\
\hline L-lisina $78 \%$ & 0,145 & 0,196 & 0,204 \\
\hline L-treonina $99 \%$ & 0,025 & 0,042 & 0,042 \\
\hline Fitase $5.000 \mathrm{ftu} / \mathrm{g}$ & 0,010 & 0,010 & 0,010 \\
\hline Cloreto de colina $70 \%$ & 0,060 & 0,047 & 0,030 \\
\hline Salinomicina sódica $12 \%$ & 0,050 & 0,050 & - \\
\hline Suplemento vitamínico ${ }^{1}$ & 0,200 & 0,160 & 0,100 \\
\hline Suplemento mineral ${ }^{2}$ & 0,100 & 0,100 & 0,100 \\
\hline Butil-hidroxi-tolueno & 0,010 & 0,010 & 0,010 \\
\hline Tratamento (inerte + vitamina) & 0,250 & 0,250 & 0,250 \\
\hline \multicolumn{4}{|l|}{ Composição nutricional } \\
\hline EMAn $(\mathrm{kcal} / \mathrm{kg})$ & 3.100 & 3.200 & 3.250 \\
\hline Proteína bruta $(\%)$ & 21,02 & 18,780 & 17,52 \\
\hline Cálcio (\%) & 0,78 & 0,65 & 0,62 \\
\hline Fósforo disponível (\%) & 0,35 & 0,30 & 0,27 \\
\hline Lisina digestível(\%) & 1,16 & 1,05 & 0,98 \\
\hline Metionina digestível(\%) & 0,53 & 0,49 & 0,45 \\
\hline Metionina+cistina $(\%)$ & 0,82 & 0,76 & 0,71 \\
\hline Treonina digestível (\%) & 0,74 & 0,68 & 0,64 \\
\hline Sódio $(\%)$ & 0,20 & 0,19 & 0,18 \\
\hline \multicolumn{4}{|l|}{ Composição nutricional analisada 3} \\
\hline Proteína bruta $(\%)$ & 20,50 & 18,70 & 17,70 \\
\hline $\mathrm{Ca}(\%)$ & 0,86 & 0,73 & 0,70 \\
\hline $\mathrm{Pt}(\%)$ & 0,63 & 0,58 & 0,55 \\
\hline
\end{tabular}

${ }^{1}$ Suplemento vitamínico (Roche/DSM). Níveis de garantia/kg do produto: vit. A - 10.000.000 UI; vit. E - $40.000 \mathrm{UI}$; vit. $\mathrm{K}_{3}-3.000 \mathrm{mg}$; vit $\mathrm{B}_{1}-2.000 \mathrm{mg}$; vit. $B_{2}-7.000 \mathrm{mg}$; vit. $B_{6}-5.000 \mathrm{mg}$; vit. $B_{12}-20.000 \mu \mathrm{g}$; ácido fólico $1.500 \mathrm{mg}$; ácido pantotênico - $15.000 \mathrm{mg}$; niacina - $50.000 \mathrm{mg}$; biotina $100 \mathrm{mg}$; selênio - $150 \mathrm{mg}$; antioxidante - $125 \mathrm{mg}$.

2 Suplemento micromineral (Roche/DSM). Níveis de garantia/kg do produto: $\mathrm{Mn}-80.000 \mathrm{mg} ; \mathrm{Zn}-80.000 \mathrm{mg}$; Fe - $60.000 \mathrm{mg}$; Cu - $10.000 \mathrm{mg}$; I - $1.000 \mathrm{mg}$; Co $-1.000 \mathrm{mg}$.

${ }^{3}$ Análises realizadas no Laboratório de Pesquisa Animal DZO/UFLA. isenta de incidência de luz solar direta e com temperatura máxima e mínima de 25 e $20^{\circ} \mathrm{C}$, respectivamente. As rações e água foram fornecidas à vontade. Foram realizadas vacinações contra a doença infecciosa da bursa (Gumboro), aos sete e aos 14 dias de idade das aves.

As temperaturas (máxima e mínima) e a umidade relativa, médias, registradas a partir de 12 dias de idade foram de $21^{\circ} \mathrm{C}, 3^{\circ} \mathrm{C}$ e $65 \%$, respectivamente

As pesagens das aves foram feitas no $21^{\circ}, 38^{\circ}$ e $45^{\circ}$ dia de idade das aves. Aos 45 dias, 400 aves, quatro por parcela, foram abatidas, seguindo as normas de abate, sendo que metade delas foi destinada à avaliação de rendimentos de cortes e a outra metade à avaliação de características intestinais.

As rações experimentais foram analisadas quanto aos teores de proteína bruta, cálcio, fósforo, colecalciferol e 25-OHD3. As análises do teor de nitrogênio das rações experimentais foram realizadas pelo conforme metodologia proposta pelo AOAC (1990).

As análises de vitamina $\mathrm{D}_{3}$ e $25-\mathrm{OHD}_{3}$ nas rações (Tabela 3) foram realizadas no Laboratório da DSM Nutritional Products (R\&D, Analytical Research CenterARC), na Suíça. Para análise de vitamina $\mathrm{D}_{3}$, adotou-se a metodologia descrita por Mattila (1995), enquanto, para $25-\mathrm{OHD}_{3}$, adotaram-se os procedimentos descritos por Hofmann et al. (2003). Nas duas metodologias, a detecção foi realizada utilizando-se cromatografia líquida de alta performance (HPLC).

As análises da altura das vilosidades e profundidade de criptas do duodeno, jejuno e íleo foram realizadas por meio de leituras de lâminas com cortes histológicos dos respectivos segmentos ao microscópio óptico com aumento de 32 vezes (abate de cinco aves por tratamento no $45^{\circ}$ dia do experimento). Após a coleta criteriosa dos segmentos do intestino, estes foram lavados em água destilada, devidamente identificados e armazenados em solução de formol tamponado a $10 \%$, para posterior confecção das lâminas histológicas segundo Junqueira \& Junqueira (1983), com adaptações descritas por Carvalho et al. (2009). A preparação das lâminas foi realizada no Laboratório de Patologia, no Departamento de Medicina Veterinária da UFLA e as leituras micrométricas, no Laboratório de Biologia Molecular, no Departamento de Biologia da UFLA.

Os dados foram submetidos à análise estatística utilizando-se o software Sistema de Analise de Variância para Dados Balanceados (Sisvar), descrito por Ferreira (2000). Realizou-se a análise de regressão para os níveis da vitamina na avaliação até 21 dias, e teste $\mathrm{F}$ para verificar possíveis diferenças entre as fontes. No período total de 
Tabela 3 - Recuperação ${ }^{1}$ de vitamina $\mathrm{D}$ em relação à quantidade suplementada (entre parênteses) nas rações

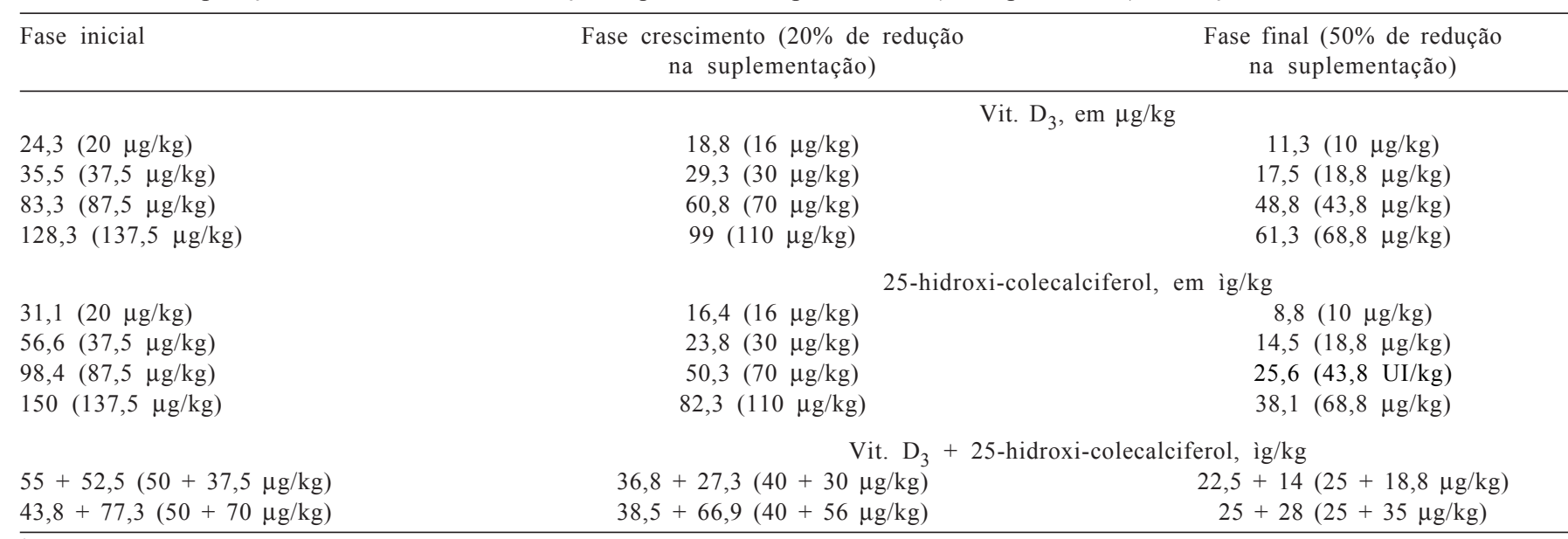

${ }^{1}$ Análises realizadas no R\&D, Analytical Research Center - ARC - DSM Nutritional Products, Suíça.

avaliação, 1 a 45 dias, foram realizados testes de média e contrastes de interesse para analisar os programas de suplementação para cada fonte de vitamina. As comparações entre os tratamentos adicionais e os demais foram realizadas por meio de contrastes, a $5 \%$ de probabilidade.

\section{Resultados e Discussão}

Não houve interação $(P>0,05)$ entre as fontes de vitamina D para o desempenho das aves na fase de 1 a 21 dias (Tabela 4) nem efeito $(P>0,05)$ das fontes de vitamina $D$ sobre o desempenho na fase inicial. Por outro lado, independentemente da fonte de vitamina D utilizada, os níveis de suplementação tiveram influência significativa no ganho de peso e na conversão alimentar. A análise de regressão revelou efeito linear $(\mathrm{P}<0,05)$, com melhorias no ganho de peso e na conversão alimentar à medida que se elevaram os níveis de vitamina $\mathrm{D}$ nas rações.

Esses resultados confirmam os obtidos por Rao et al. (2006), que verificaram aumento no ganho de peso e na melhor conversão alimentar nesta fase em frangos de corte alimentados com rações com $60 \mu \mathrm{g} / \mathrm{kg}(2.400 \mathrm{UI} / \mathrm{kg})$ e $90 \mu \mathrm{g} / \mathrm{kg}(3.600 \mathrm{UI} / \mathrm{kg})$ de vitamina D3, com níveis reduzidos de cálcio $(0,50 \%)$ e fósforo disponível $(0,25 \%)$, em detrimento de níveis de suplementação menores $(5$ e $30 \mu \mathrm{g} / \mathrm{kg}$ ). Por outro lado, os resultados encontrados na fase inicial contradizem

Tabela 4 - Desempenho das aves na fase inicial, de acordo com níveis, fontes e associação entre fontes de suplementação de vitamina D

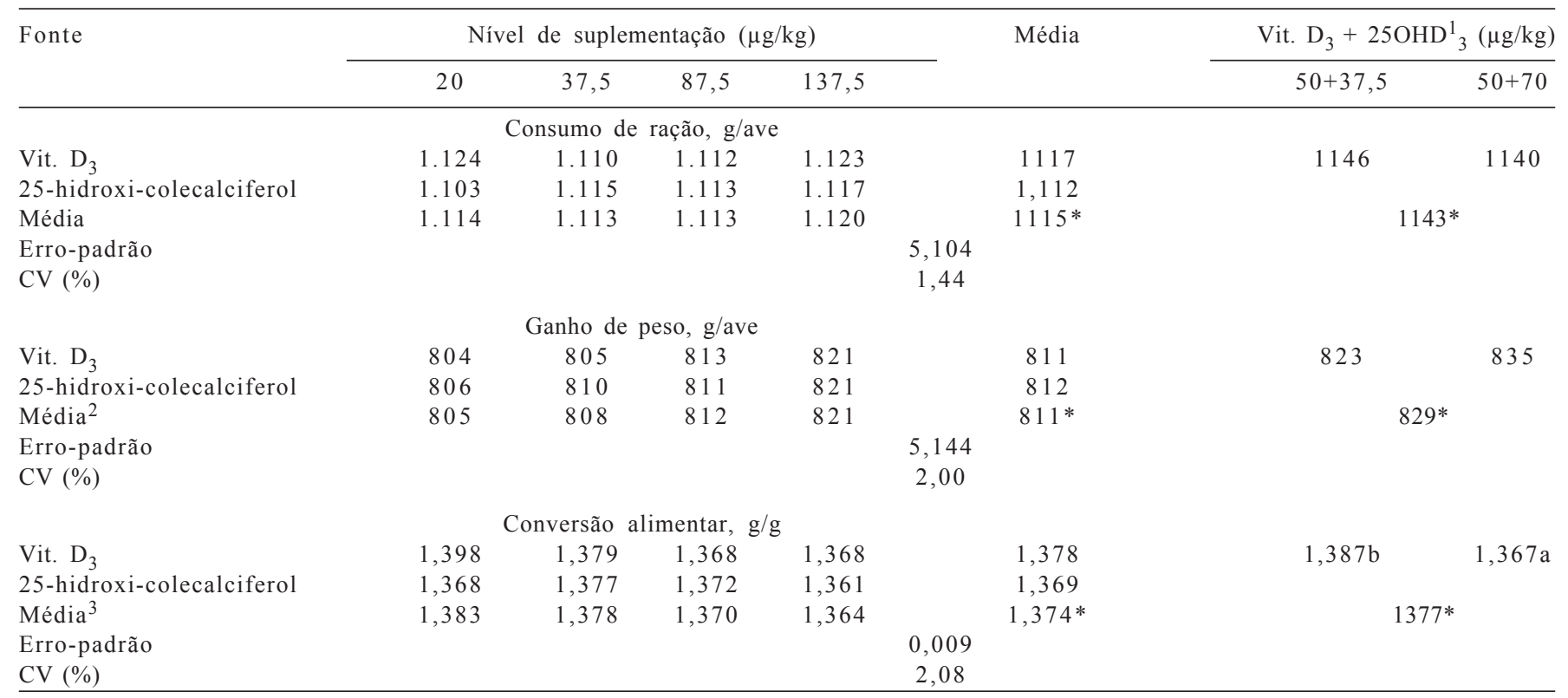

* Médias estatisticamente diferentes $(\mathrm{P}<0,01)$.

${ }_{2}^{1}$ Médias com letras diferentes na linha são estatisticamente diferentes $(\mathrm{P}<0,05)$.

2 Efeito linear: $\mathrm{GP}=802,001+0,133$ X Suplementação de vitamina $\mathrm{D} ; \mathrm{R}^{2}=97,36 \%$.

${ }^{3}$ Conversão alimentar $(\mathrm{g} / \mathrm{g})=1,3861$ - 0,00017 X suplementação de vitamina $\mathrm{D} ; \mathrm{R}^{2}=97,39 \%$. 
respostas sobre o desempenho encontradas por Mitchell (1997), que não verificou melhorias no ganho de peso e ou conversão alimentar em níveis acima de $5 \mu \mathrm{g} / \mathrm{kg}(200 \mathrm{UI} / \mathrm{kg})$ de vitamina $\mathrm{D}_{3}$.

A ausência de diferença no desempenho das aves atribuída às fontes de vitamina $\mathrm{D}$ confirma resultados obtidos por diversos autores (Zhang et al., 1997; Edwards, 2002 ; Ledwaba et al., 2003; Fritts \& Waldroup, 2005; Rao et al., 2008). Entretanto, alguns autores apontam maior efetividade da $25-\mathrm{OHD}_{3}$ sobre o desempenho de frangos, principalmente na fase inicial, quando consideram que aves apresentam maior sensibilidade às fontes e níveis de suplementação de vitamina D (Yarger et al., 1995; Fritts \& Waldroup, 2003).

As aves alimentadas com as rações contendo as duas fontes de vitamina $\mathrm{D}$ conjuntamente, nas duas proporções, apresentaram maior $(\mathrm{P}<0,001)$ consumo de ração em relação à média dos frangos provenientes da suplementação isolada de cada fonte de vitamina $\mathrm{D}$ em níveis crescentes. Como consequência, essas aves apresentaram maior $(p<0,001)$ ganho de peso nessa fase sem, no entanto, apresentar diferenças significativas $(\mathrm{P}>0,05)$ entre si.

A conversão alimentar foi pior $(\mathrm{P}<0,05)$ para os frangos alimentados com as dietas contendo a combinação de $50 \mu \mathrm{g}$ de vitamina $\mathrm{D}_{3} / \mathrm{kg}$, somada a $37,5 \mu \mathrm{g}$ de $25-\mathrm{OHD}_{3} / \mathrm{kg}$, em comparação ao segundo tratamento adicional, que apresentava mesmo nível de suplementação de vitamina $\mathrm{D}_{3}$, porém, com $70 \mu \mathrm{g}$ de $25-\mathrm{OHD}_{3} / \mathrm{kg}$.

Os efeitos positivos sobre o desempenho encontrados na primeira fase com a suplementação de vitamina $D$, independentemente da fonte, quando incorporados na fase de crescimento e associados à redução na suplementação vitamínica em $20 \%$, não foram acumulativos. Ou seja, os programas de suplementação não geraram diferenças $(\mathrm{P}>0,05)$ para as características de desempenho, indicando que níveis altos de suplementação de vitamina D não foram eficientes em melhorar o desempenho, considerando o período de 1 a 38 dias de idade das aves (Tabela 5).

No entanto, o uso combinado das duas fontes até essa fase promoveu maior ganho de peso $(\mathrm{P}<0,01)$ em comparação aos programas com fontes isoladas. Não foram observadas $(\mathrm{P}>0,05)$ diferenças entre as aves submetidas aos dois programas adicionais, na fase de 1 a 38 dias.

Os resultados constantes na literatura geralmente não revelam grandes diferenças no desempenho atribuídas à suplementação de vitamina D na fase final de criação. As exigências para máximo desempenho normalmente são menores que $25 \mu \mathrm{g} / \mathrm{kg}$ ração (Edwards et al., 1994; NRC, 1994). A fase inicial, período em que ocorre alta taxa de crescimento do tecido esquelético, além da imaturidade do trato digestório (digestão e absorção de lipídios e compostos lipossolúveis), é provavelmente mais sensível ao aumento na suplementação.

De forma semelhante aos resultados encontrados na fase de 1 a 38 dias, na avaliação em todo o período experimental (1 a 45 dias), não foram observadas diferenças $(\mathrm{P}>0,05)$ no desempenho das aves em função dos programas de suplementação de vitamina $\mathrm{D}$, independente da fonte usada neste estudo (Tabela 6). Portanto, pode-se inferir que a suplementação de $20 \mu \mathrm{g} / \mathrm{kg}$ de ração (800 UI/ $\mathrm{kg}$ ) na fase

Tabela 5 - Desempenho de frangos de corte na fase de 1 a 38 dias de idade recebendo suplementação de vitamina D

\begin{tabular}{|c|c|c|c|c|c|c|c|}
\hline \multirow[t]{2}{*}{ Fonte } & \multicolumn{4}{|c|}{ Nível de suplementação $(\mu \mathrm{g} / \mathrm{kg})$} & \multirow[t]{2}{*}{ Média } & \multicolumn{2}{|c|}{ Vit. $\mathrm{D}_{3}+25 \mathrm{OHD}_{3}^{1}(\mu \mathrm{g} / \mathrm{kg})$} \\
\hline & $20 / 16$ & $37,5 / 30$ & $87,5 / 70$ & $137,5 / 110$ & & $50+37,5$ & $50+70$ \\
\hline \multicolumn{8}{|c|}{ Consumo de ração, g/ave } \\
\hline $25-\mathrm{OHD}_{3}$ & 3.861 & 3.863 & 3.874 & 3.891 & 3.872 & \multirow{2}{*}{\multicolumn{2}{|c|}{$3.935^{*}$}} \\
\hline Média & 3.873 & 3.865 & 3.870 & 3.880 & $3.872 *$ & & \\
\hline Erro-padrão & \multicolumn{7}{|c|}{14,752} \\
\hline Vit. $\mathrm{D}_{3}$ & 2.372 & 2.365 & 2.372 & 2.396 & 2.376 & 2.430 & 2.436 \\
\hline $25-\mathrm{OHD}_{3}$ & 2.364 & 2.370 & 2.379 & 2.392 & 2.376 & & \\
\hline Média & 2.368 & 2.368 & 2.376 & 2.394 & $2.376 *$ & \multicolumn{2}{|c|}{$2.432 *$} \\
\hline Erro-padrão & \multirow{2}{*}{\multicolumn{7}{|c|}{$\begin{array}{c}18,988 \\
2,52\end{array}$}} \\
\hline CV $(\%)$ & & & & & & & \\
\hline Erro-padrão & & & & & & \multirow{2}{*}{\multicolumn{2}{|c|}{1,618}} \\
\hline CV $(\%)$ & & & & & & & \\
\hline
\end{tabular}

*Médias estatisticamente diferentes $(\mathrm{P}<0,01)$ 
Tabela 6 - Desempenho de frangos de corte na fase de 1 a 45 dias de idade das aves recebendo suplementação de vitamina D

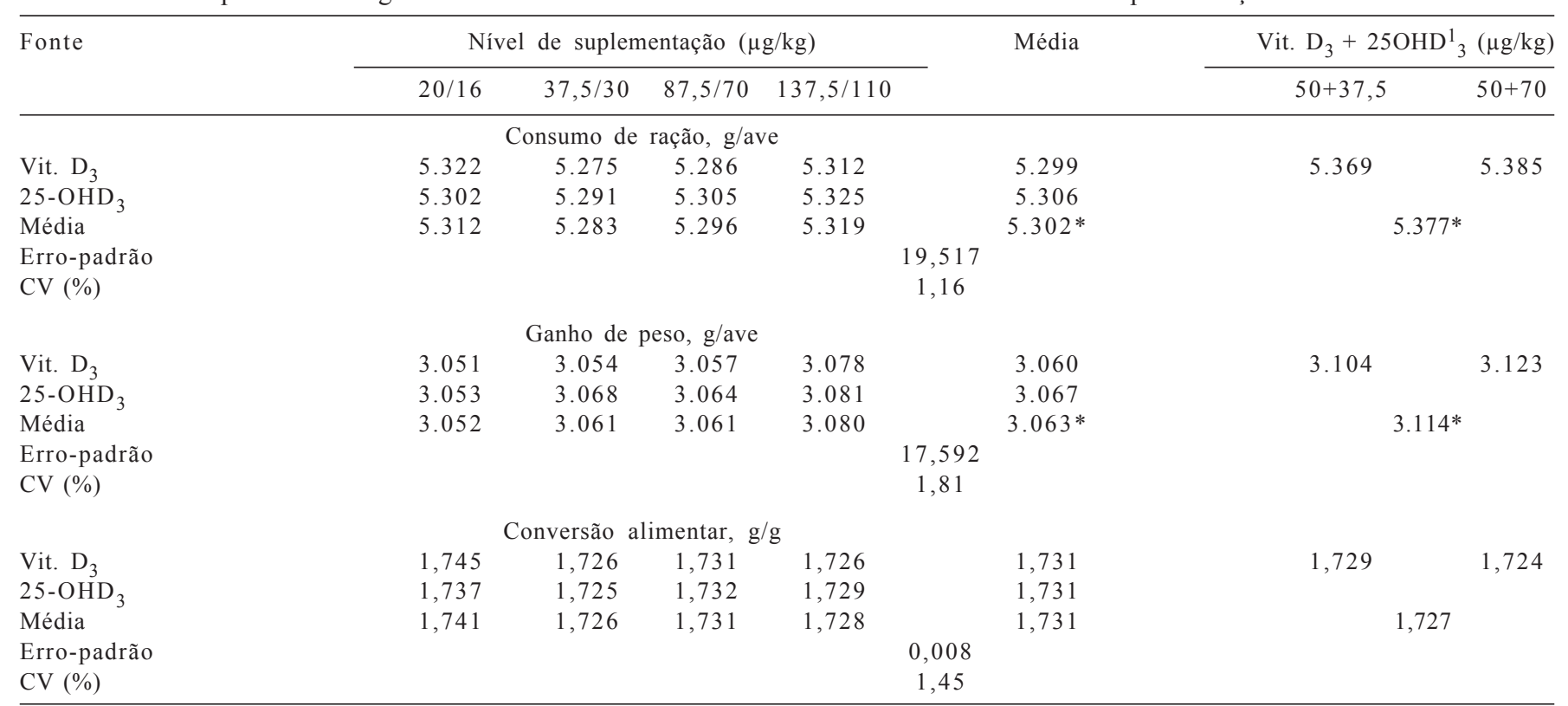

* Médias estatisticamente diferentes $(\mathrm{P}<0,001)$.

${ }_{1}$ Programas de suplementação das fontes de vitamina D: 5 - 50/40/25+37,5/30/18,8 e $6-50 / 40 / 25+70 / 56 / 35 \mu \mathrm{g} / \mathrm{kg}$ de ração.

inicial com redução de 20 e $50 \%$ nas fases de crescimento e final, respectivamente, foi suficiente para manter o desempenho adequado, quando a suplementação de vitamina $\mathrm{D}_{3}$ ou $25-\mathrm{OHD}_{3}$ for realizada isoladamente.

Os resultados obtidos seguem o mesmo padrão verificado nas fases anteriores, ou seja, a suplementação das duas fontes de vitamina $\mathrm{D}$ em diferentes proporções e em conjunto, proporcionou maiores $(\mathrm{P}<0,05)$ consumo de ração e ganho de peso no período total. Entretanto, a conversão alimentar não foi influenciada $(\mathrm{P}>0,05)$ por nenhum fator, assim como a viabilidade de criação, que não foi sensível aos programas de suplementação estudados e apresentou valor médio de $97,4 \%$.

Os resultados obtidos para desempenho estão de acordo com a maioria dos estudos que envolvem vitamina $\mathrm{D}$, ou seja, quando níveis de cálcio e/ou fósforo disponível estão dentro de limites de deficiência marginal ou atendendo às necessidades nutricionais, não são verificados efeitos diretos do incremento da suplementação de vitamina D (Mitchel et al., 1997; Zhang et al., 1997; Baker et al., 1998; Edwards, 2002; Fritts \& Waldroup, 2005). Por outro lado, respostas mais sensíveis no desempenho são observadas em condições de redução nos níveis de cálcio e fósforo (Rao et al., 2006), podendo haver recuperação do desempenho através da otimização nos processos de absorção e metabolismo desse elementos (Nahm, 2007).

Assim, a utilização das duas fontes combinadas resultou em ganho de peso $1,66 \%$ superior à média dos demais programas. Todavia, quando se compararam os tratamentos adicionais ao programa com maior nível de suplementação em cada fonte isolada $(137,5 / 110 / 68,8)$, essa diferença caiu para $1,1 \%$. É necessário ressaltar que não houve benefício do uso de altos níveis de suplementação em qualquer das fontes de vitamina $\mathrm{D}$ sobre a conversão alimentar.

Não houve interação $(P>0,05)$ entre fontes e programas avaliados para as características de carcaça (Tabela 7), porém, verificou-se aumento $(\mathrm{P}<0,05)$ no rendimento de carcaça em função das fontes de vitaminas avaliadas, ou seja, a $25-\mathrm{OHD}_{3}$ aumentou o rendimento em comparação à vitamina $\mathrm{D}_{3}$. Esses resultados corroboram aqueles observados por Korver (2005), porém, diferem dos encontrados por Angel et al. (2006). O estudo sobre os efeitos de diferentes fontes de vitamina D sobre características de carcaça ganhou força nos últimos anos e há relatos de redução nas perdas e de condenações das carcaças nos abatedouros, em decorrência da melhoria na qualidade ou densidade óssea. Assim, há muito que pesquisar sobre esses efeitos.

Não houve efeito dos fatores $(\mathrm{P}>0,05)$ sobre o rendimento de peito e coxa + sobre coxa, assim como os diferentes programas de suplementação e ou tratamentos adicionais não afetaram o rendimento de carcaça. Os resultados do rendimento de peito diferem dos encontrados por Korver (2005) e Larroude et al. (2005), que verificaram aumento no rendimento de peito em frangos de corte e no rendimento de filé de peito em perus, respectivamente, com o uso de 25-OHD3, sem apresentar explicações sobre este fato. 
Tabela 7 - Características de carcaça de frangos de corte aos 45 dias de idade recebendo suplementação de vitamina D

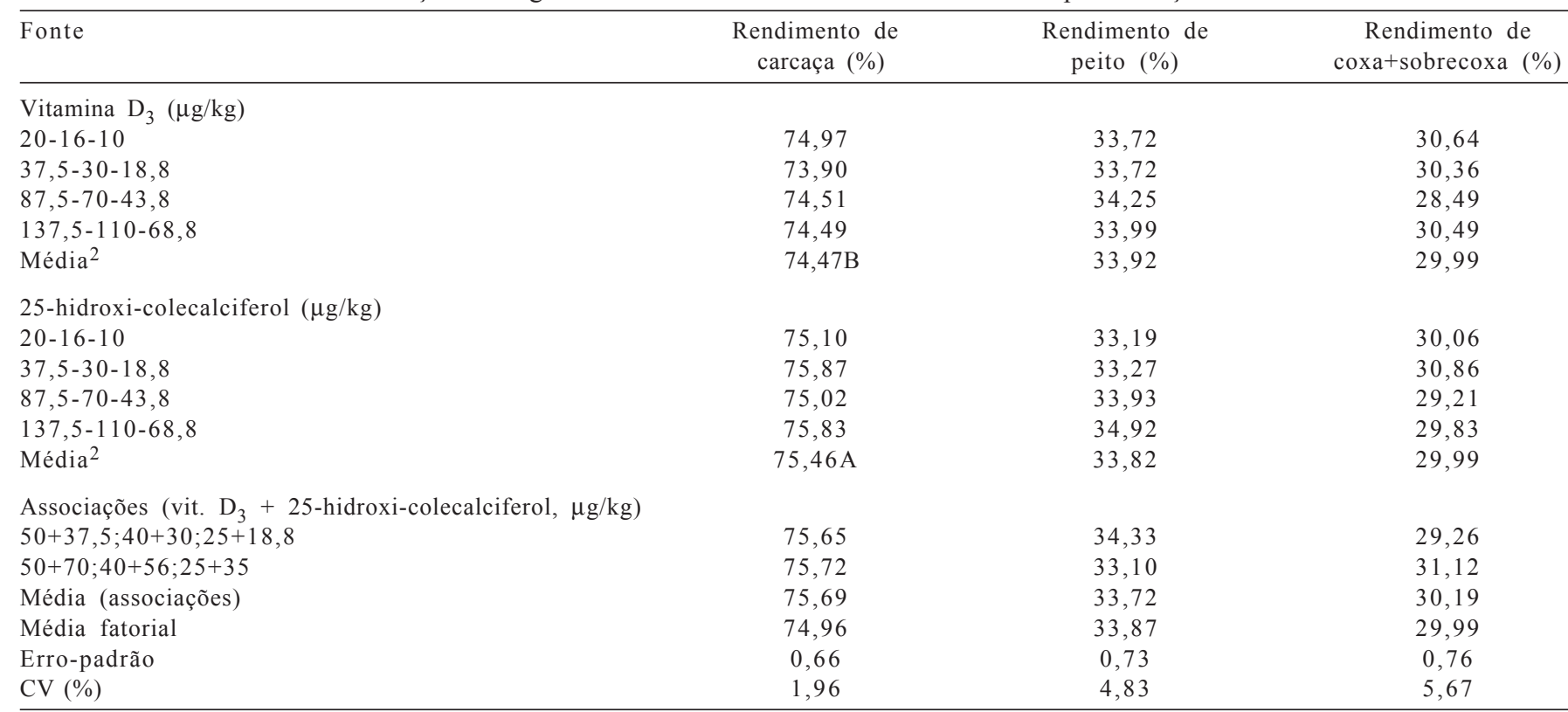

${ }^{1}$ Médias seguidas por letras diferentes na coluna diferem estatisticamente $(\mathrm{P}<0,05)$.

Tabela 8 - Características intestinais em frangos de corte aos 45 dias de idade, de acordo com fontes e associações de suplementação de vitamina D

\begin{tabular}{|c|c|c|c|}
\hline Fonte & \multicolumn{3}{|c|}{ Característica $^{1}$} \\
\hline & \multicolumn{3}{|c|}{ Duodeno } \\
\hline Média $\left(25 \mathrm{OHD}_{3}\right)$ & 1512 & 180 & 8,41 \\
\hline Média associações & 1518 & 176 & 8,65 \\
\hline Erro-padrão & 65,3 & 15,2 & 2,5 \\
\hline Média $\left(D_{3}\right)$ & 1226 & 136 & 9,06 \\
\hline Média $\left(25 \mathrm{OHD}_{3}\right)$ & 1209 & 127 & 9,55 \\
\hline Média associações & 1207 & 129 & 9,36 \\
\hline Erro-padrão & 51,1 & 12,8 & 2,5 \\
\hline $\mathrm{CV}(\%)$ & 17,8 & 19,7 & 20,8 \\
\hline Erro-padrão & 47,1 & 13,4 & 1,96 \\
\hline CV $(\%)$ & 18,8 & 20,9 & 23,8 \\
\hline
\end{tabular}

Não foram verificados $(\mathrm{P}>0,05)$ efeitos dos diferentes fatores sobre a morfologia intestinal (duodeno, jejuno e íleo). Esses resultados sugerem que os programas adotados com as duas fontes em estudo, assim como a combinação entre elas, não geraram qualquer alteração que possa ter ocasionado modificação na capacidade absortiva de nutrientes ao longo do intestino delgado, o que pode ter refletido na ausência de efeitos significativos sobre o desempenho.

\section{Conclusões}

A suplementação de vitamina D influi positivamente no ganho de peso e na conversão alimentar de frangos de corte na fase inicial, mas não tem efeitos nas outras fases de criação. A utilização conjunta das duas fontes de vitamina $\mathrm{D}$ (vitamina $\mathrm{D}_{3}$ e $25-\mathrm{OHD}_{3}$ ) melhora o desempenho de frangos de corte, independentemente da fase de criação. 
Além disso, o uso do metabólito de vitamina D 25-hidroxicolecalciferol melhora o rendimento de carcaça em relação ao uso de vitamina $\mathrm{D}_{3}$. A fonte de vitamina $\mathrm{D}$ e o nível de suplementação na ração não influenciam as características morfológicas do intestino delgado de frangos de corte.

\section{Referências}

ANGEL, R.; SAYLOR, W.W.; MITCHELL, A.D. et al. Effect of dietary phosphorus, phytase and 25-hydroxycholecalciferol on broiler chicken bone mineralization litter phosphorus, and processing yields. Poultry Science, v.85, n.7, p.1200-1211, 2006.

ASSOCIATION OF OFFICIAL ANALYTICAL CHEMISTS AOAC. Official methods of analysis. 12.ed. Washington, D.C.: 1990. 1230p.

BAKER, D.H.; BIEHL, R.R.; EMMERT, J.L. Vitamin D3 Requirement of young chicks receiving diets varying in calcium and available phosphorus. British Poultry Science, v.39, n.3, p.413-417, 1998.

CARVALHO, J.C.C.; BERTECHINI, A.G.; FASSANI, E.J. et al. Desempenho e características de carcaça de frangos de corte alimentados com dietas à base de milho e farelo de soja suplementadas com complexos enzimáticos. Revista Brasileira de Zootecnia, v.38, n.2, p.292-298, 2009.

EDWARDS JR., H.M.; ELLIOT, M.A.; SOONCHARERNYING, S. et al. Quantitative requirement for cholecalciferol in the absence of ultraviolet light. Poultry Science, v.73, n.2, p.288-294, 1994.

EDWARDS JR., H.M. Studies on the efficiency of cholecalciferol and derivatives for stimulating phytate utilization in broilers. Poultry Science, v.81, n.7, p.1026-1031, 2002.

FERREIRA, D.F. SISVAR - Sistema para análise de variância para dados balanceados: programa de análises estatísticas e planejamento de experimentos, versão 4.3. Lavras: UFLA, 2000. 66p.

FRITTS, C.A.; WALDROUP, P.W. Effect of source and level of vitamin $D$ on live performance and bone development in growing broilers. Journal Applied Poultry Research, v.12, n.1, p.45-52, 2003.

FRITTS, C.A.; WALDROUP, P.W. Comparasion of cholecalciferol and 25-hydroxychloecalciferol in broilers diets designed to minimize phosphorus excretion. Journal Applied Poultry Research, v.14, n.1, p.156-166, 2005.

HOFMANN, P.; GOESSL, R.; DENU, L. Determination of 25hidroxyvitamin D3 (Hy-D) in animal feeds using $d_{6}-25-$ hidroxyvitamin as internal standard: DSM report, $n$. 1012520, Method Report. Switzerland: DSM Nutricional Products, 2003.

JUNQUEIRA, L.C.U.; JUNQUEIRA, L.M.M.S. Técnicas básicas de citologia e histologia. São Paulo: Universidade de São Paulo, 1983. 123p.
KORVER, D. Research, analytical techniques and pratical experiences using $\mathrm{HyD}^{\mathrm{TM}}$. In: ARKANSAS NUTRITION CONFERENCE, 2005, Arkansas. Proceedings... Arkansas: [s.n.], 2005. 12p.

LARROUDÉ, P.; CASTAING, J.; HAMELIN, C. et al. Effet dune supplementation en $H_{-} D^{\circledR}$ pour deux niveaux dápports en vitamines sur les performances, le developpenment osseux et les troubles locomoteurs des dindons. Sixiemes Journees de la ReCherche Avicole, n.3, p.244-248, 2005.

LEDWABA, M.F.; ROBERSON, K.D. Effectiveness of twentyfive-hydroxycholecalciferol in the prevention of tibial dyscondroplasia in Ross cockerels depends on dietary calcium level. Poultry Science, v.82, n.11, p.1769-1777, 2003.

MATTILA, P. Analysis of cholecalciferol, ergocalciferol and their 25-hydroxylated metabolities in food by HPLC. 1995. 130f. Dissertation (Ph.D.) - University Helsinki, Helsinki, Finland.

MITCHELL, R.D.; EDWARDS JR., H.M.; MCDANIEL, G.R. The effects of ultraviolet light and cholecalciferol and its metabolites on the development of leg abnormalities in chickens genetically selected for a high and low incidence of tibial dyschondroplasia. Poultry Science, v.76, n.2, p.346-354, 1997.

NAHM, K.H. Efficient phosphorus utilization in poultry feeding to lessen the environmental impact of excreta. World's Poultry Science Journal, v.63, n.4, p.625-654, 2007.

NATIONAL RESEARCH COUNCIL - NRC. Nutrient requirements of poultry. 9.ed. Washington, D.C.: National Academic Science, 1994. 155p.

RAO, S.V.R.; RAJU, M.V.L.N.; PANDA, A.K. Effect of high concentrations of cholecalciferol on growth, bone mineralization and mineral retetion in broiler chicks fed suboptimal concentrations of calcium and nonphytate phosphorus. Journal of Applied Poulry Research, v.15, n.4, p.493-501, 2006.

RAO, S.V.R.; RAJU, M.V.L.N.; PANDA, A.K. et al. Effect of surfeit concentrations of vitamin D3 on performance, bone mineralization and mineral retetion in broiler chicks. Journal of Poultry Science, v.45, n.1, p.25-30, 2008.

ROSTAGNO, H.S.; ALBINO, L.F.T.; DONZELE, J.L. et al. Composição de alimentos e exigências nutricionais de aves e suínos (Tabelas Brasileiras). Viçosa, MG: Universidade Federal de Viçosa, 2005. 186p.

SCHOUTEN, N.A.; TEIXEIRA, A.S.; FREITAS, R.T.F. et al. Níveis de cálcio em rações de frangos de corte na fase inicial suplementadas com fitase. Revista Brasileira de Zootecnia, v.32, n.5, p.1190-1197, 2003.

YARGER, J.G.; QUARLES, C.L.; HOLLIS, B.W. et al. Safety of 25-hydroxycholecalciferol in poultry rations. Poultry Science, v.74, n.9, p.1437-1446, 1995.

ZHANG, X.; LIU, G.; MCDANIEL, G.R. et al. Response of broiler lines selected for tibial dyschondroplasia incidence to supplementary 25-hydro xycholecalciferol. Journal Applied Poultry Research, v.6, n.4, p.410-416, 1997. 